\title{
Influence of Laser Irradiation Low Intensity on Reparative Osteogenesis and Angiogenesis Under Transosseous Osteosynthesis
}

\author{
Yuri Mikhailovich Iryanov* \\ Russian Ilizarov Scientific Center Restorative Traumatology and Orthopedics, Russian Federation
}

\author{
*Correspondence to \\ Yuri Mikhailovich Iryanov, MD; \\ Russian Ilizarov Scientific Center \\ Restorative Traumatology and \\ Orthopedics, Russian Federation. \\ Tel: +7-3522466316, + 98- \\ 44225624; \\ Fax: +91-8025734656; \\ Email: irianov@mail.ru
}

Published online 18 July 2016

\begin{abstract}
Introduction: The use of non-medicinal facilities of correcting processes for various pathological conditions is one of the most urgent problems of modern medicine. The purpose of the work is to study the efficiency of low-intensive of infrared laser irradiation in promoting reparative osteogenesis and angiogenesis during fracture treatment under transosseous osteosynthesis with a qualitative and quantitative morphological analysis.

Methods: A tibial fracture was modeled experimentally in rats from control and experimental groups, then repositioning and fixation of fragments performed. The fracture zone of the experimental group animals was exposed to pulsed infrared laser irradiation of low intensity. The animals from control group underwent irradiation simulation. The operated bones were investigated using $\mathrm{x}$-ray, light and electron microscopy, x-ray electron probe microanalysis.

Results: The sessions of laser irradiation decreased inflammatory process severity, activated fibrillogenesis and angiogenesis, accelerated the compactization of newly formed bone tissue, and enhanced its maturity degree while primary healing occurred in the fracture. Conclusion: Laser therapy of fracture zone ensures the formation of regenerated bone and fragment union within earlier periods.

Keywords: reparative osteogenesis; angiogenesis; laser irradiation; fracture; transosseous osteosynthesis.
\end{abstract}

\section{Introduction}

The use of non-medicinal facilities of correcting the processes of reparative regeneration for various pathological conditions is one of the most urgent problems in modern medicine. Laser therapy of different types is widely used in clinical practice including traumatology and orthopaedics for treating the locomotorium injuries and diseases, and it is aimed to provide the anesthetizing, anti-edematous, anti-inflammatory and trophic-stimulating effects. ${ }^{1-4}$ Besides, it ensures the decrease in medicinal burden for the patient. The treatment with laser beams is comfortable, non-invasive, aseptic, painless, harmless, and controllable. ${ }^{5,6}$ The low-energy laser has a multifactorial effect on the organism. ${ }^{7,8}$ The enzymatic activity, as well as the rate of oxygen consumption by tissues increases, also the level of peroxide lipid oxidation decreases, oxidation-restoration processes activates, and the concentration of adaptive hormones increases. ${ }^{9,10}$ At the same time, the mechanism of laser therapeutic effects is largely unclear, and the doses are selected empirically. Both experimental-and-morphological studies in this field and information on infrared laser effect on reparative osteogenesis morphology are not numerous, ${ }^{1,2,11}$ thereby evidencing the problem relevance, as well as the necessity of performing the present study. The purpose of the work is to study the efficiency of low-intensive of infrared laser irradiation in promoting reparative osteogenesis and angiogenesis during fracture treatment under transosseous osteosynthesis with a qualitative and quantitative morphological analysis.

\section{Methods}

We performed the experiments using 32 Wistar pubertal male rats of 340-390-g body weight in the control and experimental groups (16 animals each).

We modeled a tibial fracture in the shaft middle third in a closed way under general anesthesia, performed reposition and fixation of fragments using the device worked out by us (Iryanov YM, Naumov EA, Iryanova TY; Russian Ilizarov Scientific Center Restorative Traumatology and Orthopaedics; Device for osteosynthesis of small bones. Russia patent 113651. 2012 February 12). One day after surgery the animals of the experimental group underwent low-energy pulsed infrared laser irradiation using a therapeutic laser device (laser therapy apparatus "Uzor A-2K," country of origin: Russia), wavelength within the near-infrared range $(0.89 \pm 0.02 \mu \mathrm{m})$, pulse frequency $-150 \mathrm{~Hz}$, power $-4 \mathrm{~W}$, pulse duration - 110-160 
ns. The irradiation of the fracture zone was performed in pulse mode locally for 10 minutes. The distance between the emitter and the skin was $1 \mathrm{~mm}$. The sessions of irradiation were repeated after one day and performed at day 7 and 14 after surgery. The animals of the control group underwent irradiation simulation where the therapeutic laser device was switched off. Eight animals were used for each time point. We took $\mathrm{x}$-rays immediately after surgery and in the process of treatment.

We fixed the operated bones in phosphate buffer paraformaldehyde and glutaraldehyde $2 \%$ solution ( $\mathrm{pH} 7.4$ ), and further fixed the regenerated bone pieces in $1 \%$ solution of osmium tetroxide for electron microscopy. The specimens were dehydrated in a series of ethanol increasing concentrations, in $100 \%$ acetone, and then embedded in paraffin (after decalcification) and in araldite (without decalcification). The paraffin-embedded histotopographic longitudinal sections were stained with hematoxylin-eosin, and with picrofuchsin by Van Gieson. The morphological analysis and photomicrography of the histological preparations were performed using Stemi 2000C microscope complete with AxioCam ERc $5 s$ digital camera and Zen blue (Carl Zeiss Micro Imaging GmbH, Germany) software. The araldite-embedded bones were studied in regard to the characteristic $\mathrm{x}$-ray irradiation of calcium using INCA-200 Energy x-ray electron probe microanalyzer (Oxford Instruments Analytical, England). The osteogenesis process was determined by the content of bone structures in the intermediate zone of the regenerated bone. The organ specificity and the degree of newly formed bone tissue maturity were evaluated by the index of compactness (bone tissue/non-mineralized structure content ratio). We sawed the blocks in the zone of regenerated bone to obtain the specimens for electron microscopy, prepared ultra-thin sections of 70-90-nm thickness using LKB-8800 ultramicrotome (Sweden), contrasted them with uranyl acetate and lead citrate solutions, and studied them using JEM-2010 (Jeol, Japan) transmission electron microscope under the accelerating voltage of $80 \mathrm{kV}$. Then we treated the specimens with $2 \%$ sodium ethyolate solution (to remove araldite from the surface), sprayed them with platinum and palladium alloy (in 1:3 ratio) in $I B-6$ ion vacuum sprayer (Eico, Japan), and studied them in secondary electrons under the accelerating voltage of $20 \mathrm{kV}$ using JSM-840 scanning electron microscope (Jeol, Japan). The number of vessels and their lumen diameter were determined by scanning electronograms in the section area unit of $0.01 \mathrm{~mm}^{2}$ for x1000 instrumental magnification.

The results of the quantitative investigations were processed by the methods of variation statistics. The significance of differences in the compared parameters was calculated using Student $t$ test. Differences were considered significant for the level of $P<0.05$.

\section{Results}

Clearly marked diastasis was observed by x-rays in the operated bone of the animals from control group 7 days after the fracture occurrence; moreover, delicate hardly detectable cloud-like shadows of the regenerated bone appeared near the ends of fragments. Early signs of formation of low-contrast periosteal stratifications of little extent were observed. Microscopy revealed foci of alternatively destructive changes in the zone of bone fragments, as well as areas of organizing hematoma infiltrated by lymphocytes, neutrophil granulocytes, monocytes, mast cells and macrophages. Bone debris, filaments, fibers and clots of fibrin were revealed in the intermediate and endosteal zones of the regenerated bone; little-differentiated connective tissue with signs of edema was formed. The intermediate zone of the regenerated bone was mostly replaced by small islets of poorly vascularized little-differentiated and scarring granulation tissue as well as bundles of collagen fibers. The structures of newly formed bone tissue were not revealed in the intermediate zone. Isolated sinusoids with moderate ecstasies, parietal micro thrombi, diapedesis of erythrocytes and leukocytes were observed in the regenerated bone. Perivascular cells were sporadic (Figure 1A). A thin layer of newly formed bone trabeculae was formed along the endosteal surface of fragments. There was no endosteal and periosteal bone union.

Callus located all over the bone diameter was revealed by $\mathrm{x}$-rays 7 days after fracture occurrence in the animals of the experimental group. Contrast periosteal stratifications appeared. The dense shadows of the regenerated bone directed towards the regenerated bone of the fragmental ends from the periosteal and endosteal surfaces. Polynuclear macrophage cells, degranulated neutrophils, and mast cells were revealed in the regenerated bone, and fibrin clots were calcified. Active osteogenesis was noted, expressed as mass proliferation of osteogenic cells of the periosteum and endosteum, as well as formation of significant amounts of periosteal and endosteal expansions which partially or completely overlapped the diastasis. Newly formed blood vessels were located in the intermediate zone of the regenerated bone, numerous anastomosing trabeculae of reticulofibrous bone tissue appeared, which grew towards each other and formed stratifications on the ends of fragments from the periosteal and endosteal surfaces. Granulation tissue was vascularized with multiple capillaries filling all the space between newly formed bone structures and fragments. Signs of the increased permeability of the endothelial layer were registered in the vessels of the microcirculatory bed that was evidenced by the multiplicity of micropinocytic vesicles, the dissociation of endothelial contacts, as well as by appearing interendothelial pores and slots on the luminal surface. Simultaneously, capillary buds were revealed as endovasal endothelial outgrowths at different stages of canalization with typical imbricate surface micro relief formed by flat marginal portions of adjacent endotheliocytes (Figure 1C), as well as growing capillary terminals (Figure 1B, 1D) that evidenced the active processes of reparative angiogenesis.

The results of electron probe microanalysis (Table 1) evidenced osteogenesis activation and increase in the matu- 
rity degree of newly formed bone tissue in the regenerated bones of the animals of the experimental group compared with the control one. Thus, the content of bone tissue in the regenerated bones (177.09\%) exceeded the parameters in the control group, and the index of compactness increased more than twice (222.727\% higher). In the animals of the experimental group, angiogenesis was activated and evidenced by $173.3 \%$ increase in the number of vessels, as well as by $75.9 \%$ decrease in their diameter compared with control.

The primary regenerated bone was formation and signs of the initial stage of periosteal union appeared 14 days after surgery in the control group of animals (Figure 2A), with the prevalence of fibrous cartilage between fragments, as well as dense fibrous connective tissue. Fracture union occurred by secondary type with formation of, mainly, periosteal callus, represented by a network of weaving bone trabeculae of different maturity degrees which formed as a result of endochondral osteogenesis. The forming primary osteons were determined.

In the experimental group of animals, bone fragments were connected by periosteal-and-endosteal bone structures in the form of vertical brackets 14 days after surgery. Periosteal stratifications of $1.5-2 \mathrm{~mm}$ in length compactized and integrated the fragmental ends as a fusiform coupling. The regenerated bone in the intermediate zone presented as spongy bone tissue tightly soldered with fragmental ends and overlapping the diastasis (Figure 2B, $2 \mathrm{C}, 2 \mathrm{D})$. The formation of new bone cortical layer was observed. Fracture healing was of primary type. Fragmental ends were connected by the secondary osteons of lamellar bone tissue of different maturity degrees with compactization scenes. In the intertrabecular spaces numerous blood vessels were located in the lumen of which the endovasal endothelial outgrowths were detected. Mast
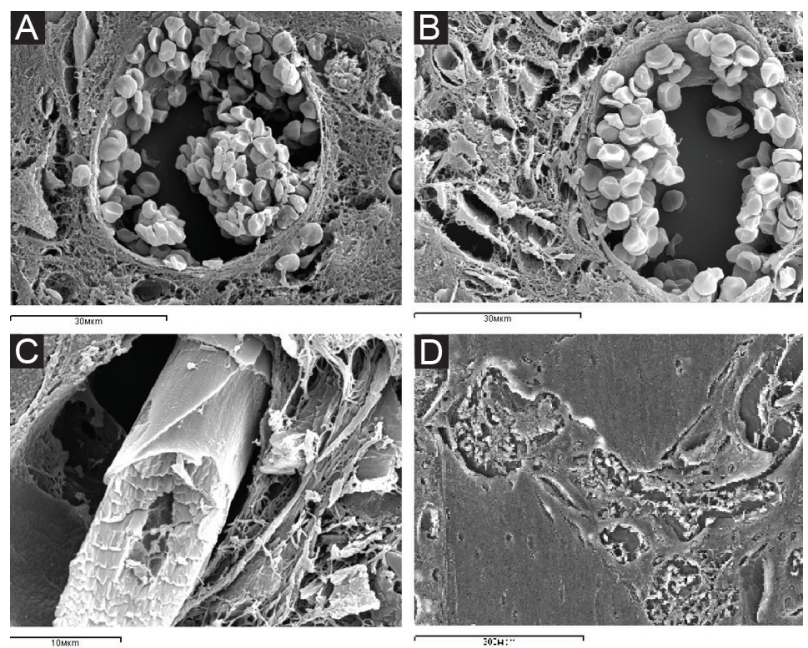

Figure 1. Blood Vessels in the Intermediate Zone of Regenerated Bones 7 Days After Fracture Occurrence. (A) a sinusoid in the regenerated bone of the animal from control group; (B), (C), (D) newly formed capillary terminals (arrows) in the regenerated bone of the animals from experimental group; (A), (B), (C) scanning electron microscopy, (d) an ultra-thin section, transmission electron microscopy, magnification x 8000 . cells of fusiform and sprout-like shapes at different stages of degranulation were located in the perivascular space. Functionally active osteoclasts localized on the periosteal and endosteal surfaces, as well as significant stratifications of newly formed bone trabeculae surrounded by numerous large osteoblasts were noted. According to the data of electron probe microanalysis, 14 days after fracture occurrence and after six sessions of exposure to laser irradiation, the content of bone tissue in the regenerated bones increased $202.86 \%$, the index of compactness increased $347.5 \%$, and the number of vessels increased $294.5 \%$ with $60.8 \%$ decrease in their diameter compared with the parameters in the animals of control group (Table 1).

\section{Discussion}

Clinical, experimental and morphological studies of GA Ilizarov et $\mathrm{al}^{12-14}$ not only proved a quick bone recovery process under transosseous osteosynthesis, but also laid the foundation for the further search for ways to correct reparative processes in the regenerated bone. According to our study, one of the approaches to optimization of reparative osteogenesis and angiogenesis is exposure to low-intensity infrared laser radiation. The maximum of laser irradiation transmission by the skin is within the wavelength range of $0.8-1.2 \mu \mathrm{m}$, that is why the exposure to infrared laser beams is especially effective, because they penetrate well into the damaged soft tissues and reach the zone of bone fracture, the regenerated bone is therefore subjected to direct irradiation impact. ${ }^{3}$ The stimulating effect of laser radiation on reparative bone formation and angiogenesis may be not direct, but mediated and implemented with the participation of complex systems of autocrine, paracrine, neuroendocrine and immune regulation. Thus the primary cellular target in regenerated bone as in soft tissue damage, are mast cells, with stimulation
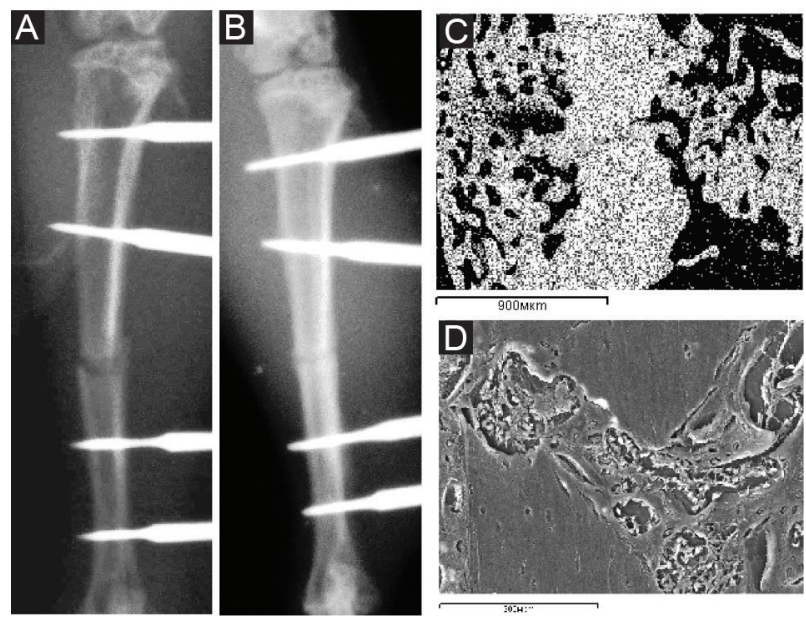

Figure 2. The Rat Tibias 14 Days After Surgery. (A) the bone of the animal from control group; (B), (C), (D) the bones of the animals from experimental group; (A), (B) x-rays; (C) the image of fracture zone in the characteristic $x$-ray irradiation of calcium, the map of $x$-ray electron probe microanalysis; (D) the image of fracture zone in secondary electrons, scanning electron microscopy, magnification x 2000 . 
Table 1. Characteristics of Osteogenesis and Angiogenesis in the Intermediate Zone of the Regenerated bone in the Animals of Control and Experimental Groups $(\mathrm{M} \pm \mathrm{m})^{\mathrm{a}}$

\begin{tabular}{|c|c|c|c|c|}
\hline \multirow{3}{*}{ Parameters } & \multicolumn{4}{|c|}{ Period of the Experiment, Days } \\
\hline & \multicolumn{2}{|c|}{7} & \multicolumn{2}{|c|}{14} \\
\hline & Control & Experimental & Control & Experimental \\
\hline Bone tissue, \% & $18.38 \pm 0.90$ & $32.55 \pm 1.51^{b}$ & $28.67 \pm 1.52$ & $58.16 \pm 2.11^{b}$ \\
\hline Index of compactness & $0.22 \pm 0.01$ & $0.49 \pm 0.02^{b}$ & $0.40 \pm 0.02$ & $1.39 \pm 0.07^{b}$ \\
\hline Number of vessels & $1.65 \pm 0.07$ & $2.86 \pm 0.15^{b}$ & $2.23 \pm 0.14$ & $4.56 \pm 0.17^{b}$ \\
\hline Vascular lumen diameter, $\mu \mathrm{m}$ & $42.75 \pm 2.05$ & $32.45 \pm 1.51^{b}$ & $49.65 \pm 2.05$ & $30.18 \pm 1.09^{b}$ \\
\hline
\end{tabular}

${ }^{a}$ The data are presented as the Mean $(\mathrm{M})$, representativeness error $(\mathrm{m})$.

${ }^{\mathrm{b}}$ Significant changes comparing with the parameters of the animals from control group, $P<0.05$.

of secretory activity which is an important mechanism of amplification of the system response to the effects of laser radiation, as evidenced by the literature and our data. ${ }^{15}$ The analysis of the data we obtained evidences the fact that reparative osteogenesis during bone fracture healing via transosseous osteosynthesis under the effect of infrared laser irradiation occurs considerably more actively than in control group. This manifests itself in decreased severity of inflammatory process, intensified microcirculation and fibrillogenesis, as well as acceleration of newly formed bone tissue reorganization and compactization, with increase in its maturity degree, in earlier regenerated bone formation and bone union. The intermediate regenerated bone is formed just 7 days after surgery with laser therapy, and periosteal, intermediate and endosteal bone union is determined after 14 days. The fracture healing is of the primary type. After laser therapy sessions, prolonged capillary dilatation is observed in the regenerated bone, as well as endothelium-dependent vasodilatation and intense capillarogenesis. Wherein, the expression of endotheliocyte migration phenotype is promoted, as well as formation of endothelial outgrowths in vascular lumen which form the sprouts of capillaries spreading along "maternal" vessels over long distances without the resistance of perivascular tissue structures. This method of formation and growth of capillaries directly into the lumen of the preexisting vessels, designated by us as endovasal capillarogenesis, first described in the healing of bone injuries, ${ }^{15-17}$ is one of the types of the initial stage of the regeneration of angiogenesis, which provides accelerated regeneration and oriented growth of new vessels.

\section{Conclusion}

The findings revealed a possible mechanism of laser irradiation effect at the level of the whole organism, and they confirm the efficiency of its use in clinical practice at the early stage of the patient rehabilitation under transosseous osteosynthesis.

\section{Ethical Considerations}

Keeping, surgical interventions and euthanasia of the animals were performed according to the guidelines of the RF Ministry of Health and those of "European Convention for the Protection of Vertebrate Animals used for Ex- perimental and other Scientific Purposes.”

\section{Conflict of Interests}

Authors declare that they have no conflict of interests.

\section{Acknowledgements}

We are thankful to the staff of our institutions for the assistance in carrying out the experiments and care for the animals at all the stages of our work.

\section{References}

1. Baibekov IM, Khanapiiaev UK. The healing of leg bone fractures in rats, and some immunological parameters for magnetic laser therapy and osteosynthesis according to Ilizarov. Bull Exp Biol. 2001;4:472.

2. Lamnitskii NI, Bineshevskii EV. Mechanisms of promoting reparative osteogenesis with laser irradiation. Stomatology. 1993;5:18.

3. Nuzov BG, Nuzova OB. Structural-and-functional evaluation of impacting the combined use of Miliacyl and laser irradiation in trophic ulcer treatment. Morfologiia. 2003;124(5):31-33.

4. Osipenko AV, Zhukov PV. The potential of low-intensive laser irradiation in complex treatment of long bone fractures using transosseous osteosynthesis method. Genij Ortop. 2007;1:125.

5. Bjordal JM, Couppé C, Chow RT, Tunér J, Ljunggren EA. A systematic review of low level laser therapy with locationspecific doses for pain from chronic joint disorders. Aust $J$ Physiother. 2003;49(2):107-116. doi:10.1016/s00049514(14)60127-6.

6. Holden PK, Li C, Da Costa V, et al. The effects of laser irirradiation of cartilage on chondrocyte gene expression and the collagen matrix. Lasers Surg Med. 2009;41(7):487491. doi:10.1002/lsm.20795.

7. Bondarenko OG, Popov GK. The effect of low-intensive laser irradiation on eosinophils isolated from peripheral blood. Bull Exp Biol. 2004;11:577.

8. Solov'eva LI, Kozel' AI, Popov GK. The mechanism of the effect of low-intensive laser irradiation on a cell. Bull Exp Biol. 1999; 10: 397.

9. Mordon S. Cartilage reshaping by laser in stomatology and maxillofacial surgery. Rev Stomatol Chir Maxillofac. 2004; 105(1): 42-49.

10. Foulad A, Ghasri P, Garg R, Wong B. Stabilization of costal cartilage graft warping using infrared laser irirradiation in a porcine model. Arch Facial Plast Surg. 2010;12(6):405- 
411. doi:10.1001/archfacial.2010.93.

11. Mavrich VV. Some details of the growth and chemical composition of different skeletal bones of white rats under the impact of X-ray and laser irradiation. Morphology. 1999;4:57.

12. Ilizarov GA, Iryanov YM. Features osteogenesis under tensile stress. Bull Exp Biol. 1991;2:194.

13. Shevtsov VI, Iryanov YM. Osteogenesis and angiogenesis during distraction osteosynthesis. Bull Exp Biol. 1995;7: 95. doi:10.1001/archfacial.2010.93.

14. Iryanov YM, Iryanova TY. Reparative bone formation at the lengthening of limbs under transosseous distraction osteosynthesis. Morphology. 2003;3: 83.
15. Iryanov YM, Kiryanov NA. Reparative bone formation and angiogenesis under conditions of low-intensity electromagnetic radiation of extremely high frequency. Bull Med Sci. 2015;3: 334.

16. Iryanov YM, Dyuryagina OV. Effect of the local granulation tissue focus formed in the medullary cavity on reparative osteogenesis. Bull Exp Biol. 2014;1:121. doi:10.1007/ s10517-014-2503-3.

17. Iryanov YM, Popkov AV, Antonov NI. Morphological details of reparative osteogenesis under transosseous osteosynthesis and intramedullary insertion of wires with hydroxyapatite coating. Morphology. 2014;4:53. 